\title{
APLICAÇÃO DO ÍNDICE DE QUALIDADE DE ATERROS DE RESÍDUOS (IQR) EM ÁREA DE DISPOSIÇÃO DE RESÍDUOS SÓLIDOS URBANOS DO MUNICÍPIO DE RIACHO FRIO - PI
}

Application index quality of iandfill waste (iqr) in area layout solid waste urban the municipality in Riacho Frio - PI

\author{
RÓGERS PINHEIRO GAMA DA SILVA ${ }^{1}$ \\ MORECKS FERREIRA DE AMORIM ${ }^{2}$ \\ CHARLES ALVES NOBRE ${ }^{3}$ \\ TANCIO GUTIER AILAN COSTA ${ }^{4}$ \\ ISRAEL LOBATO ROCHA ${ }^{5}$
}

RESUMO: Atualmente o crescimento da população e a forte industrialização, contribuíram para o rápido aumento de resíduos sólidos, acelerando o processo contínuo de deterioração ambiental que gera sérias implicações na qualidade de vida da sociedade. No Brasil, é recente a preocupação com o gerenciamento adequado, tratamento e disposição final dos resíduos sólidos urbanos. $\mathrm{O}$ atual sistema de coleta e disposição final de resíduos da cidade de Riacho Frio - PI vem funcionando com dificuldade operacional, onde pode classificar o local de depósito como lixão a céu aberto. Desta forma, é necessário que os municípios brasileiros busquem a adequação às normativas vigentes dos sistemas de coleta, transporte, tratamento e disposição final dos resíduos, viabilizando a sustentabilidade ambiental urbana. O estudo teve como objetivo, aplicar o índice de qualidade de aterro de resíduo - (IQR) para avaliar o sistema de disposição final do município de Riacho Frio - PI, a fim de aprimorar os mecanismos de controle sanitário ambiental. Para tanto, são apresentadas as atividades de concepção metodológica, levantamentos de dados, sistematização de informações, conforme proposta pela CETESB (Companhia Ambiental do Estado de São Paulo) nas quais atendem as condições mínimas ambientais e de operacionalização como: distância de corpos hídricos e áreas residenciais, cercamento e vias de

\footnotetext{
${ }^{1}$ Graduando do Curso Tecnologia em Gestão Ambiental pelo Instituto Federal de Educação, Ciência e Tecnologia do Piauí - Campus Corrente, E-mail: rogergama10@hotmail.com

${ }^{2}$ Graduando do Curso Tecnologia em Gestão Ambiental pelo Instituto Federal de Educação, Ciência e Tecnologia do Piauí - Campus Corrente.

${ }^{3}$ Graduando do Curso Tecnologia em Gestão Ambiental pelo Instituto Federal de Educação, Ciência e Tecnologia do Piauí - Campus Corrente.

${ }^{4}$ Graduando do Curso Tecnologia em Gestão Ambiental pelo Instituto Federal de Educação, Ciência e Tecnologia do Piauí - Campus Corrente.

${ }^{5}$ Professor do Curso Tecnologia em Gestão Ambiental pelo Instituto Federal de Educação, Ciência e Tecnologia do Piauí - Campus Corrente, E-mail: israel.lobato@ifpi.edu.br
} 
acesso. O valor do IQR obtido foi de 1,84 mostrando que as condições da área são inadequadas, em razão do valor ser menor que 6,0. Isto revela que a área não atende de forma satisfatória muitos itens dentro das características locais, infraestrutura e condições operacionais.

Palavras-Chave: Índice de qualidade de resíduos; disposição final dos resíduos sólido; lixão.

\section{ABSTRACT}

Currently population growth and strong industrialization, contributed to the rapid increase in solid waste, accelerating the ongoing process of environmental degradation that create serious implications for the quality of life of society. In Brazil, recent concern for the proper management, treatment and disposal of municipal solid waste. The current collection system and final disposal of waste in the city of Riacho Frio - PI has worked with operational difficulty, where you can sort the storage area to dump the open. Thus, it is necessary that municipalities seek adaptation to current regulations of collection systems, transport, treatment and disposal of waste, enabling the urban environmental sustainability. The study aimed to apply the waste landfill quality index - (IQR) to evaluate the final disposal system Riacho Frio municipality - PI in order to improve the mechanisms of environmental health control. Therefore, the activities of methodological design are presented, data surveys, systematization of information, as proposed by CETESB (Environmental Company of the State of São Paulo) in which meet the environmental and operational minimum conditions such as distance from water bodies and areas residential, fencing and access roads. The value obtained IQR was 1.84 showing that area conditions are inadequate, because the value is less than 6.0. This shows that the area does not meet the satisfaction of many items within the local characteristics, infrastructure and operational conditions.

KEYWORDS: Index of quality waste; disposal end waste solid; dumping ground.

\section{INTRODUÇÃO}

Atualmente o crescimento da população e a forte industrialização, contribuíram para o rápido aumento de resíduos sólidos, acelerando o processo contínuo de deterioração ambiental que gerem sérias implicações na qualidade de vida da sociedade. No Brasil, é recente a preocupação com o gerenciamento adequado, tratamento e disposição final dos resíduos sólidos urbanos.

A quantidade de resíduos sólidos produzida atualmente no mundo é muito grande e o seu mau gerenciamento, além de provocar gastos financeiros significativos e sérios danos ao meio ambiente, pode comprometer a saúde e o bem-estar da população. 
De acordo com a NBR 10.004 da ABNT (Associação Brasileira de Normas Técnicas) existem diferentes tipos de disposição dos resíduos sólidos: aterro sanitário, aterro controlado e lixão.

Os lixões são locais onde o lixo coletado é lançado diretamente sobre o solo sem qualquer controle e sem quaisquer cuidados ambientais, poluindo tanto o solo, quanto o ar e as águas subterrâneas e superficiais das vizinhanças.

A disposição inadequada dos resíduos sólidos promove a contaminação do solo, do ar e das águas superficiais e subterrâneas, além da proliferação de vetores de doenças, influenciando negativamente a qualidade ambiental e a saúde da população (LEITE et al., 2003). Os "lixões" não possuem nenhum tipo de controle, quer quanto ao tipo de resíduos recebidos, quer em relação às medidas de segurança necessárias, para minimizar ou evitar emissões de poluentes para o meio ambiente.

Os resíduos assim lançados acarretam problemas à saúde pública, como proliferação de vetores de doenças (moscas, mosquitos, baratas, ratos), geração de maus odores e, principalmente, a poluição do solo e das águas superficiais e subterrâneas.

Desta forma, o presente estudo teve como objetivo aplicar o índice de qualidade de aterro de resíduo - (IQR) para avaliar o sistema de disposição final do município de Riacho Frio - PI, a fim de aprimorar os mecanismos de controle sanitário ambiental.

\section{MATERIAIS E MÉTODOS}

\section{Área de Estudo}

O município de Riacho Frio localiza-se no Extremo Sul do Piauí, com área de 2.222,1 km² de acordo com o Instituto Brasileiro de Geografia e Estatística (IBGE), o município contava om uma população estimada de 4.255 habitantes (IBGE, 2014). Possui uma distância de cerca de 870 $\mathrm{km}$ de Teresina, capital do estado do Piauí (Figura 1). Encontra-se inserido no bioma cerrado, onde o clima que predomina é o tropical, com duas estações bem definidas: uma chuvosa, entre outubro e abril, e outra seca, entre maio e setembro. 


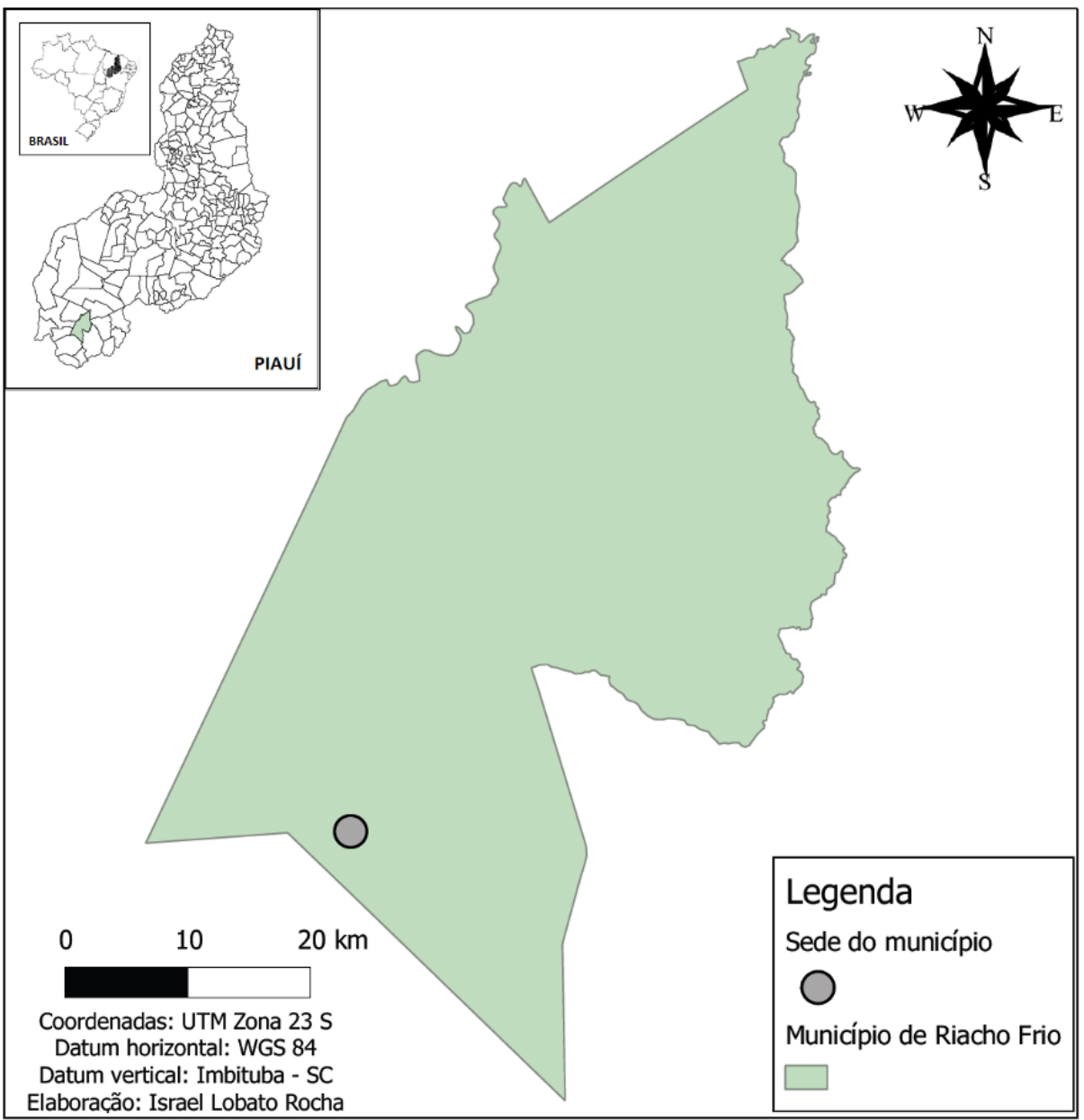

Figura 1 - Localização da área de estudo. Fonte: Rocha et al., 2013.

\section{Procedimentos metodológicos}

As informações para o cálculo do IQR, foram coletadas por meio de visitas técnicas ao local de disposição final do município Riacho Frio-PI, localizado próximo à rodovia PI 255. O estudo foi realizado utilizando-se a metodologia adotada pela CETESB, que consiste no preenchimento do "check list" padronizado, considerando as características do local, infraestrutura implantada, condições operacionais. Essas 3 partes do questionário do IQR é constituídas por 41 parâmetros cujas as pontuações variam de 0 a 10.

Todas essas etapas possuem pesos diferentes. As pontuações apresentam como base matemática, a soma do subtotal de cada item, o total, dividido por 13, determina o valor do IQR, conforme classificação conforme a Tabela 1 : 
Tabela 1 - Enquadramento dos valores do IQR referentes aos locais de disposição final dos resíduos sólidos.

\begin{tabular}{c|c}
\hline IQR & ENQUADRAMENTO \\
\hline 0,0 a 6,0 & Condições inadequadas \\
\hline 6,1 a 8,0 & Condições controladas \\
\hline 8,1 a 10 & Condições adequadas \\
\hline
\end{tabular}

Os resultados enquadram os vazadouros em inadequados, se o IQR verificado for inferior a 6; em condições controlados, quando IQR está entre 6 e 8; e, adequados, se o índice estiver entre 8 e 10 .

\section{RESULTADOS E DISCUSSÕES}

A adoção de indicadores, sub-índices e índices, podem servir como referenciais para o planejamento e melhorias operacionais dos serviços de limpeza urbana, nos municípios, pois demonstram as influências dos sistemas, nas áreas de saúde pública, desenvolvimento econômico, social e ambiental. O questionário padronizado com a pontuação atribuída a cada subitem, bem como os valores obtidos no IQR do lixão a céu aberto do município de Riacho Frio apresentam os resultados conforme as Tabelas 2 e 3 .

Tabela 2 - Questionário padronizado com os parâmetros do IQR.

\begin{tabular}{|c|c|c|c|c|}
\hline \multicolumn{2}{|c|}{ Item } & Subitem & Avaliação & Pontuação \\
\hline \multirow{10}{*}{\multicolumn{2}{|c|}{ 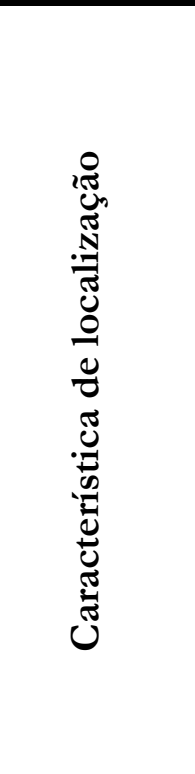 }} & Capacidade de suporte do solo & Inadequada & 0 \\
\hline & & Proximidade de núcleos habitacionais & Longe $>500 \mathrm{~m}$ & 5 \\
\hline & & Proximidade de corpos de água & Longe $>200 \mathrm{~m}$ & 3 \\
\hline & & Profundidade de lençol freático & Maior 3m & 4 \\
\hline & & Permeabilidade do solo & Media & 2 \\
\hline & & Material para recobrimento & Nenhuma & 0 \\
\hline & & Qualidade do material para recobrimento & Ruim & 0 \\
\hline & & $\begin{array}{l}\text { Condições de sistemas viário trânsito de } \\
\text { acesso }\end{array}$ & Regulares & 2 \\
\hline & & Isolamento visual da vizinhança & Bom & 4 \\
\hline & & Legalidade de localização & Local proibido & 0 \\
\hline$=-$ & $\sigma$ & Cercamento da área & Não & 0 \\
\hline
\end{tabular}




\begin{tabular}{|c|c|c|c|}
\hline & Portaria/guarita & Não & 0 \\
\hline & Impermeabilização da base do aterro & Não & 0 \\
\hline & Drenagem de chorume & Inexistente & 0 \\
\hline & Drenagem de águas pluviais definitivas & Inexistente & 0 \\
\hline & Drenagem de águas pluviais provisórias & Inexistente & 0 \\
\hline & Trator de esteira compatível & Inexistente & 0 \\
\hline & Outros equipamentos & Não & 0 \\
\hline & Sistema de tratamento de chorume & Insuf./ inexist. & 0 \\
\hline & Acesso á frente de trabalho & Ruim & 0 \\
\hline & Vigilantes & Não & 0 \\
\hline & Sistema de drenagem de gases & Inexistente & 0 \\
\hline & Controle e recebimento de cargas & Não & 0 \\
\hline & Monitoração de águas subterrâneas & Inexistente & 0 \\
\hline & Atendimento a estipulação de projeto & Não & 0 \\
\hline \multirow{15}{*}{ 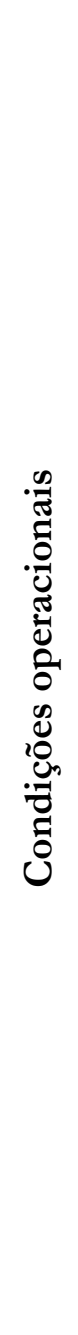 } & Aspecto geral & Ruim & 0 \\
\hline & Ocorrência de lixo descoberto & Sim & 0 \\
\hline & Recobrimento do lixo & Inexistente & 0 \\
\hline & Presenças de urubus & Sim & 0 \\
\hline & Presenças de moscas em grande quantidade & $\operatorname{Sim}$ & 0 \\
\hline & Presença de catadores & Não & 0 \\
\hline & Criação de animais & Sim & 0 \\
\hline & Descarga de resíduos de serviço de saúde & Sim & 0 \\
\hline & Descarga de resíduos industriais & Não / adequada & 4 \\
\hline & $\begin{array}{l}\text { Funcionamento de drenagem pluvial } \\
\text { definitiva }\end{array}$ & Inexistente & 0 \\
\hline & $\begin{array}{c}\text { Funcionamento de drenagem pluvial } \\
\text { provisória }\end{array}$ & Inexistente & 0 \\
\hline & Funcionamento de drenagem de chorume & Inexistente & 0 \\
\hline & $\begin{array}{l}\text { Funcionamento do sist.. de tratamento de } \\
\text { chorume }\end{array}$ & Inexistente & 0 \\
\hline & $\begin{array}{l}\text { Funcionamento do sist.. de monitorização } \\
\text { de águas subterrâneas }\end{array}$ & Inexistente & 0 \\
\hline & Eficiência da equipe de vigilância & Ruim & 0 \\
\hline
\end{tabular}




\begin{tabular}{c|c|c|c}
\hline & Manutenção dos acessos internos & Ruim & 0 \\
\hline
\end{tabular}

Tabela 3 - Valores do IQR do local de disposição final dos RSU de Riacho Frio-PI.

\begin{tabular}{l|c}
\hline Item & Subtotal \\
\hline Características do local & 20 \\
\hline Infraestrutura implantada & 0 \\
\hline Condições operacionais & 4 \\
\hline IQR & $\mathbf{1 , 8 4}$ \\
\hline
\end{tabular}

Em se tratando das características do local do lixão municipal, no que tange a proximidade com núcleo habitacional, este não se enquadra nos parâmetros estabelecidos pela Norma Técnica da ABNT - NBR 13.896 (ABNT, 1997), em relação à localização (Tabela 4), salvo pelo distanciamento de hidrografias secundárias $(1,5 \mathrm{Km})$. O mesmo localiza-se a uma distância de 490 metros da zona urbana e 220 metros das vias de acesso (Figura 2).

Tabela 4 - Distâncias mínimas permitidas para os locais de disposição final dos RSU.

\begin{tabular}{c|c}
\hline Fator & Distância mínima \\
\hline Áreas urbanas & $2.000 \mathrm{~m}$ \\
\hline Hidrografia & $200 \mathrm{~m}$ \\
\hline Vias de acesso & $200 \mathrm{~m}-500 \mathrm{~m}$ \\
\hline
\end{tabular}

Fonte: ABNT, 1997 apud DALMAS et al, 2011.

Observando os resultados obtidos do IQR ao local de disposição final dos RSU da cidade de Riacho Frio, o lixão enquadra-se como local em condições inadequadas para receber resíduos sólidos.

Para a destinação final dos resíduos sólidos, duas alternativas podem ser propostas para pequenos e médios municípios: implantação de centros de resíduos no município ou implantação de centros de resíduos compartilhados por mais de um município. Uma boa gestão de resíduos sólidos não consiste somente em uma disposição final ambientalmente adequada do lixo, ou seja, é preciso ações conjuntas que integrem educação ambiental, redução na geração dos resíduos, substituição tecnológica por matérias primas menos impactantes (COSTA; CRESPO, 2012) e, por fim, a reciclagem.

\section{CONCLUSÕES}


O deposito inadequado de resíduos sólidos em áreas sob condições irregulares, especificamente no que se refere aos lixões a céu aberto, acaba por gerar danos aos ecossistemas naturais, assim como influencia diretamente sobre a qualidade de vida da população. Neste contexto, problemas como proliferação de vetores de doenças, e poluição de solo e da água, acabam afetando a sadia qualidade de vida da população submetida a viver nas proximidades do lixão a céu aberto, como é o caso do município de Riacho Frio, Piauí.

Desta forma, torna-se importante a adoção de medidas que visem reduzir os problemas relacionados com a disposição inadequada dos resíduos sólidos, como a criação de um aterro sanitário, forma ambientalmente adequada para destinar os resíduos sólidos, ou até mesmo um centro de resíduos sólidos no município e associações destes com outros municípios vizinhos.

\section{REFERÊNCIAS}

ASSOCIAÇÃO BRASILEIRA DE NORMAS TÉCNICAS. NBR 13.896: Aterros de Resíduos não Perigosos. Critérios para Projeto, implantação e operação. Rio de Janeiro, 1997.

COMPANHIA DE TECNOLOGIA E SANEAMENTO AMBIENTAL DO ESTADO DE SÃO PAULO - CETESB. Relatório Ambiental Preliminar (RAP), Roteiros básicos. São Paulo: CETESB, 1998.

DALMAS, F. B. et al. Geoprocessamento aplicado à gestão de resíduos sólidos na UGRHI-11 Ribeira de Iguape e Litoral Sul. São Paulo, UNESP, Geociências, v. 30, n. 2, p. 285-299, 2011.

INSTITUTO BRASILEIRO DE GEOGRAFIA E ESTATÍSTICAS - IBGE. IBGE Cidades Riacho Frio (Piaui). Disponível em: http://cidades.ibge.gov.br/. Acesso: 20 de ago. 2015. 